\title{
Functional Outcomes Following Total Knee Arthroplasty Without Patellar Resurfacing: A Minimum Two-Year Follow-Up Retrospective Cohort Study
}

Daniel Li ${ }^{1}$, Andrew S. Bi ${ }^{2}$, Sahej S. Samra ${ }^{3}$, Nehal S. Samra ${ }^{3}$, Decheng Wu ${ }^{4}$, Yuangzheng Ma 5

1. Orthopedics, The Ohio State University Wexner Medical Center, Columbus, USA 2. Orthopaedic Surgery, New York University Langone Health, New York, USA 3. Orthopaedic Surgery, Northwestern University Feinberg School of Medicine, Chicago, USA 4. Medicine, Chinese Academy of Sciences, 301 PLA Military Hospital, Beijing, CHN 5. Orthopaedic Surgery, Second Affiliated Hospital of Guangzhou Medical University, Guangzhou, CHN

Corresponding author: Sahej S. Samra, sahej.samra@northwestern.edu

\begin{abstract}
This paper seeks to address the effectiveness of total knee arthroplasty (TKA) when performed without patellar resurfacing. The objective of this article is to investigate the effect of total knee arthroplasty without patellofemoral resurfacing on postoperative outcome.
\end{abstract}

All patients with degenerative knee osteoarthritis (OA) that underwent TKA without patellar resurfacing were included in the study. The clinical data of 163 patients, including 98 females and 65 males with a mean age of 63 years (range 54-78 years) were retrospectively analyzed from April 2008 to April 2011.

Intraoperative cartilage degeneration according to Outerbridge classification criteria was as follows: 22 cases of grade I, 38 cases of grade II, 64 cases of grade III, 39 cases of grade IV. There were no significant differences in gender, age, and side differences between the patients at all levels $(P>0.05)$. The duration of tourniquet use and related complications were recorded. Knee function was assessed using the American Knee Society Scoring System (KSS) and the patellar score (PS). Patient satisfaction and knee pain were assessed by the pain visual analog scale (VAS). The evaluation was conducted using routine X-ray film to observe the position of the prosthesis and the patella. Statistical analysis used included a comparison between groups by analysis of variance (ANOVA) using the Student-Newman-Keuls (SNK) test and comparison of grade data using the rank-sum test.

Received 06/24/2020 Review began 01/14/2021 Review ended 06/21/2021 Published 06/29/2021

\section{(c) Copyright 2021}

$\mathrm{Li}$ et al. This is an open access article distributed under the terms of the Creative Commons Attribution License CC-BY 4.0., which permits unrestricted use, distribution, and reproduction in any medium, provided the original author and source are credited.
The average tourniquet time was 125 minutes, with a range of 90-150 minutes. All the incisions healed with primary intention without early complications. All patients were followed for two to five years with an average of 3.6 years. At six months and at the last follow-up, the KSS and PS scores were significantly higher than those before surgery $(\mathrm{P}<0.05)$. There was no significant difference between the sixth month and the last follow-up $(\mathrm{P}>0.05)$. There were significant differences in preoperative KSS and PS scores between patients with different grades of cartilage degeneration $(\mathrm{P}<0.05)$, but there was no significant difference at the last follow-up $(\mathrm{P}>0.05)$. At the last follow-up, seven patients had persistent anterior knee pain, five patients had mild pain, and two patients had moderate pain according to the VAS assessment criteria. Patient satisfaction evaluation was as follows: 90 patients were very satisfied, 66 patients were satisfied, five patients were uncertain, and two patients were unsatisfied. There were no significant differences in satisfaction and knee pain between patients with different grades of patellofemoral degeneration $(P>0.05)$.

In conclusion, at six months and at the last follow-up, outcome measures for patients were significantly higher than before surgery for TKA without the use of patellar resurfacing and the majority of patients were satisfied with the outcome of the procedure. TKA continues to be a successful procedure without the use of patellar resurfacing.

Categories: Orthopedics

Keywords: knee osteoarthritis, patellar resurfacing, post-operative outcomes

\section{Introduction}

Total knee arthroplasty (TKA) is one of the most effective methods for the treatment of knee arthritis. However, there is currently no consensus on whether patellar resurfacing significantly affect postoperative outcomes [1]. A considerable percentage (6\%-25\%) of patients undergoing primary TKA with and without patellar resurfacing report postoperative anterior knee pain, and there are notable differences related to the surgical options between countries. For example, in North America, Denmark, and Australia, 90\%, 80\%, and $60 \%$ of surgeons resurface the patella, respectively, while only $2 \%$ perform the procedure in Sweden and Norway [2].

While some studies have shown that the incidence of anterior knee pain and reoperation rate in patients 
with patellar resurfacing is lower than in patients without patellar resurfacing [3], complications following patellar replacement such as periprosthetic fracture, avascular necrosis, aseptic loosening, and postoperative infection are higher [4-6]. Studies also demonstrate that the incidence of patellofemoral clunk in the resurfaced group was significantly greater than in the group without patellar resurfacing [7]. Advocates of leaving the patella alone argue that patellar resurfacing provides no advantages in functional outcomes, reoperation rate, or overall healthcare cost, and is instead linked to more complications [8]. Conversely, nonresurfacing appears to be associated with a higher likelihood of anterior knee pain, readmission, and additional interventions, but it is also related to a higher number of reoperations, which may be related to the surgeon's tendency to revise a non-resurfaced patella [5,9]. However, these past studies are not able to clarify the discrepancy in patellar resurfacing rates among surgeons in North America and Europe [10]. Most studies have suggested that the replacement of the patella during TKA should be based on the individual patient's condition and the severity of patellofemoral degeneration [11-12], but this recommendation continues to lack clear selection criteria and treatment guidelines.

The purpose of this study is to investigate postoperative outcomes of TKA when performed without patellofemoral arthroplasty. The authors hypothesize that the overall outcomes of procedures without patellofemoral arthroplasty will be satisfactory.

\section{Materials And Methods \\ Patient selection criteria}

We retrospectively analyzed 163 non-resurfaced patellas in TKAs performed at the Affiliated Tumor Hospital of Xinjiang Medical University from April 2008 to April 2011. Inclusion criteria were as follows: a diagnosis of degenerative osteoarthritis of the knee, surgeries were performed at the Center of Orthopedics at the 309th Hospital of PLA, Beijing, 100091 in China by surgeons in the same faculty practice group, unilateral primary TKAs using the Gemini MK II cemented prosthesis (Link, Germany), no patellar resurfacing, routine functional exercise was performed after surgery, and follow-up time was greater than or equal to two years. Exclusion criteria included: indications for TKA other than osteoarthritis, knee varus or valgus deformity greater than $15^{\circ}$, ipsilateral hip joint disease, low back pain, or psychiatric disorders, or non-compliance with the investigators. The study was approved by the Ethics Committee of the Affiliated Tumor Hospital of Xinjiang Medical University.

\section{Surgical methods}

Under general anesthesia, patients were positioned supine, with a tourniquet. A standard medial parapatellar approach was performed for exposure, and then all bony cuts, ligament balancing, implant insertion, and cement fixation were performed according to the Gemini MK II cemented prosthesis operative technique guide. At the end of cementing, the tourniquet was deflated and hemostasis was achieved with electrosurgery. Analgesia was injected around the joint using previously described "cocktails" following routine surgery [13]. These "cocktails" included $30 \mathrm{ml}$ total of intraarticular injection consisting of $20 \mathrm{ml}$ of $0.5 \%$ bupivacaine local anesthetic, $1 \mathrm{ml}$ of morphine $(10 \mathrm{ml}), 0.3 \mathrm{ml}$ of epinephrine, and $9.7 \mathrm{ml}$ of normal saline $(0.9 \% \mathrm{NaCl})$. A standard layered closure was performed, covered with a dressing and cotton pad, and pressure-bandaged.

\section{Perioperative management}

Prophylactic second-generation cephalosporin antibiotics were administered 30 minutes to one hour prior to anesthetic induction and continued for 24 hours following surgery. Appropriate postoperative deep venous thrombosis (DVT) prophylaxis of the lower extremities was given to all patients. Analgesia was maintained with celebrex for four weeks postoperatively. On postoperative day one, patients were encouraged to perform high-straightening quadriceps functional exercises, muscle isometric contraction exercises, and continuous passive motion (CPM) functional exercises.

\section{Data collection}

The tourniquet time during operation and the occurrence of related complications were recorded. Knee joint function was assessed using the American Knee Society Scoring System (KSS) [13] and the patellar score (PS) [14]. Patient satisfaction and knee pain were assessed by the pain visual analog scale (VAS). Satisfaction and pain were divided into four grades. Satisfaction was divided into very satisfied ( $>8$ points), satisfactory (6-8 points), uncertain (3-5 points), and unsatisfactory ( 0 - 2 points). The pain was divided into no pain ( 0 - 2 points), mild pain (3-5 points), moderate pain (6-8 points), and severe pain (> 8 points). Routine X-ray films were taken according to the X-ray film evaluation standard established by the American Knee Association [15]. The position of the patella was evaluated according to the patellofemoral joint physical examination protocol delineated by Lester et al. [16].

\section{Statistical methods}

All patient data were analyzed using SPSS Inc. (Version 16.0, Chicago, IL). The measurement data were expressed as mean \pm standard deviation. The comparison between groups was analyzed by analysis of 


\section{Cureus}

variance (ANOVA). The comparison between the two groups was performed using the Student-NewmanKeuls (SNK) test. The comparison of grade data was performed using the rank-sum test. The test level was $\alpha=0.05$.

\section{Results}

\section{Patient information}

A total of 163 patients met the selection criteria for inclusion in the study. This group contained 65 males and 98 females whose ages ranged from 54 to 78 years, with an average of 63 years. The body mass index (BMI) ranged from $19 \mathrm{~kg} / \mathrm{m} 2$ to $30 \mathrm{~kg} / \mathrm{m} 2$ with an average of $24 \mathrm{~kg} / \mathrm{m} 2$. All patients suffered from degenerative osteoarthritis of one knee, including 54 cases of the left knee and 109 cases of the right knee. The course of disease ranged from two to eight years with an average of five years. All patients had pain around the knee joint and experienced limited mobility, and in all cases, more conservative treatment was found to be ineffective. Upon admission, the examination gave the following: knee joint range of motion (ROM) arcs ranged from 50 to $100^{\circ}$, with an average of $80^{\circ}$. According to the Outerbridge grading criteria [17], the intraoperative patellar cartilage degeneration was as follows: 22 cases of grade I, 38 cases of grade II, 64 cases of grade III, and 39 cases of grade IV. There were no significant differences in gender, age, and side differences between the patients at all levels $(\mathrm{P}<0.05)$ (Table 1$)$.

\begin{tabular}{|c|c|c|c|c|c|}
\hline Group & $\mathbf{n}$ & Gender (male : female) & Age (years) & Side (left : right) & Disease duration (years) \\
\hline Grade I & 22 & $9: 13$ & $64.2 \pm 4.3$ & $7: 15$ & $3.1 \pm 2.4$ \\
\hline Grade II & 38 & $15: 23$ & $64.8 \pm 4.2$ & $12: 26$ & $3.6 \pm 2.7$ \\
\hline Grade III & 64 & $26: 38$ & $66.7 \pm 3.4$ & $23: 41$ & $4.8 \pm 3.1$ \\
\hline Grade IV & 39 & $15: 24$ & $67.1 \pm 4.5$ & $12: 27$ & $5.7 \pm 1.3$ \\
\hline Statistic & & $X^{2}=0.05, P=0.87$ & $F=3.43, P=0.24$ & $X^{2}=2.23, P=0.57$ & $F=9.87, P=0.02$ \\
\hline
\end{tabular}

TABLE 1: Comparison of preoperative general data among patients with different grades of cartilage degeneration

\section{Functional assessment}

All patients underwent primary TKAs. Tourniquets were used for 90 to 150 minutes with an average of 125 minutes. No early complications occurred. All patients were followed up for two to five years with an average of 3.6 years. At six months and at the last follow-up, the KSS and PS scores were significantly higher than those before surgery $(\mathrm{P}<0.05)$. There was no significant difference between the follow-up at six months and the last follow-up $(\mathrm{P}>0.05)$ (Table 2). There were significant differences in preoperative KSS and PS scores between patients with different grades of patellar cartilage degeneration $(\mathrm{P}<0.05)$, but there was no significant difference at the last follow-up ( $>>0.05$ ) (Table 3). At the last follow-up, seven patients (4.3\%) had persistent anterior knee pain. According to the VAS assessment criteria, five patients had mild pain and two patients had moderate pain. Among them, four patients had abnormal patellar position without special treatment. The other patients had no anterior knee pain. In the evaluation of patient satisfaction, 90 cases were very satisfied, 66 cases were satisfied, five cases were uncertain, and two cases were unsatisfied; overall satisfaction was $95.7 \%$. There was no significant difference in the satisfaction of patients with different patellar cartilage degeneration grades and knee pain $(P>0.05)$ (Table 4). 


\section{Cureus}

Time

Preoperative

Six months after operation

Last follow-up

Statistic
KSS

$84.7 \pm 9.8^{\dagger}$

$156.5 \pm 8.7^{\star}$

$168.6 \pm 7.8^{*}$

$F=6.45, P=0.00$
PS

$11.5 \pm 3.3^{\dagger}$

$25.4 \pm 5.3^{\star}$

$26.5 \pm 3.5^{\star}$

$F=8.92, P=0.00$

TABLE 2: Comparison of American Knee Society Scoring System (KSS) and patellar scores (PS) between pre- and post-operation $(n=163, \bar{x} \pm s)$

${ }^{*}$ Compared with preoperative value, $\mathrm{P}<0.05 ;{ }^{\dagger}$ compared with the value at 6 months after operation, $\mathrm{P}<0.05$

\begin{tabular}{|c|c|c|c|c|c|c|c|}
\hline \multirow[b]{2}{*}{ Group } & \multicolumn{3}{|c|}{ KSS } & \multicolumn{3}{|l|}{ PS } & \multirow[b]{2}{*}{ Statistic } \\
\hline & $\mathrm{n}$ & Preoperative & Last follow-up & Statistic & Preoperative & Last follow-up & \\
\hline Grade I & 22 & $84.9 \pm 7.6$ & $164.7 \pm 14.5$ & $\mathrm{t}=3.21, \mathrm{P}=0.00$ & $11.4 \pm 4.3$ & $26.2 \pm 3.2$ & $t=8.74, P=0.00$ \\
\hline Grade II & 38 & $82.3 \pm 11.2$ & $166.1 \pm 11.3$ & $\mathrm{t}=6.87, \mathrm{P}=0.00$ & $10.5 \pm 3.4$ & $26.1 \pm 3.1$ & $\mathrm{t}=9.32, \mathrm{P}=0.00$ \\
\hline Grade III & 64 & $78.4 \pm 9.2$ & $168.7 \pm 15.6$ & $\mathrm{t}=5.97, \mathrm{P}=0.00$ & $9.3 \pm 3.5$ & $27.1 \pm 4.1$ & $\mathrm{t}=11.56, \mathrm{P}=0.00$ \\
\hline Grade IV & 39 & $74.4 \pm 6.4$ & $167.3 \pm 13.7$ & $t=11.17, P=0.00$ & $8.3 \pm 4.7$ & $26.3 \pm 3.2$ & $t=13.78, P=0.00$ \\
\hline Statistic & & $F=4.35, P=0.02$ & $F=2.43, P=0.42$ & & $F=5.66, P=0.02$ & $F=2.36, P=0.84$ & \\
\hline
\end{tabular}

TABLE 3: Comparison of American Knee Society Scoring System (KSS) and patellar scores (PS) among patients with different grades of cartilage degeneration at pre- and post-operation $(\bar{X} \pm s)$

\begin{tabular}{|c|c|c|c|c|c|c|c|c|c|}
\hline \multirow[b]{2}{*}{ Group } & \multirow[b]{2}{*}{$\mathbf{n}$} & \multicolumn{4}{|l|}{ Satisfaction } & \multicolumn{4}{|c|}{ Anterior knee pain } \\
\hline & & Very satisfied & Satisfied & Not certain & Not satisfied & No & Mild & Moderate & Severe \\
\hline Grade I & 22 & 8 & 13 & 1 & 0 & 21 & 1 & 0 & 0 \\
\hline Grade II & 38 & 19 & 17 & 1 & 1 & 36 & 1 & 1 & 0 \\
\hline Grade III & 64 & 43 & 18 & 2 & 1 & 61 & 2 & 1 & 0 \\
\hline Grade IV & 39 & 20 & 18 & 1 & 0 & 38 & 1 & 0 & 0 \\
\hline Statistic & & \multicolumn{4}{|c|}{$\mathrm{X}^{2}=4.32, \mathrm{P}=0.09$} & \multicolumn{4}{|c|}{$\mathrm{X}^{2}=0.36, P=0.10$} \\
\hline
\end{tabular}

TABLE 4: Comparison of satisfaction and anterior knee pain among patients with different grades of cartilage degeneration at last follow-up

\section{Radiographic evaluation}

At postoperative day three, radiographic examination showed a femoral angle of $96.5^{\circ} \pm 2.4^{\circ}$, a tibial angle of $89.7^{\circ} \pm 0.7^{\circ}$, a posterior tilt angle of the tibial plateau of $87.3^{\circ} \pm 1.4^{\circ}$, and a femoral flexion angle of $1.1^{\circ} \pm$ $0.7^{\circ}$. All were within normal ranges. At the last follow-up, no osteolysis, periprosthetic fractures, or loosening of the prosthesis were observed in this group. There were no radiographic indications of osteonecrosis or fracture. In four cases, the patellar tracking trajectory was poor. One of the patellas was displaced $3 \mathrm{~mm}$ to the lateral side, and the third patella was tilted $5^{\circ}$ to $7^{\circ}$ laterally (average $5.7^{\circ}$ ). All four patients had different degrees of knee pain. 


\section{Discussion}

Overall, at six months and at the time of the last follow-up, outcomes for patients undergoing TKA without the use of patellar resurfacing were significantly improved than before surgery, and the majority of patients were satisfied with procedural outcomes. At six months and at the last follow-up, the KSS and PS scores were significantly higher than those before surgery, but there was no significant difference between the sixth month and the last follow-up. Moreover, there were significant differences in preoperative KSS and PS scores between patients with different grades of cartilage degeneration, but there were no significant differences at the last follow-up. At the last follow-up, the patient satisfaction rate was $95.7 \%$, and the anterior knee pain rate was $4.3 \%$. The KSS and PS scores were significantly higher than those before surgery, and the postoperative outcomes were similar among patients with varying degrees of patellar cartilage degeneration.

Historically, in the early stages of TKAs, patellar resurfacing was rarely performed by most surgeons. However, as the incidence of postoperative anterior knee pain began to increase in TKA patients, more and more surgeons began to perform patellofemoral arthroplasty under the assumption that it improved postoperative outcomes. Interestingly, due to this surge in patella replacement surgery, complications associated with patella replacement and associated revision surgery have increased [18]. To this end, some scholars believe that the patella should be selectively replaced according to the individual characteristics of the patient and the degree of degeneration of the cartilage and that only patients with Outerbridge grade IV patellar defects are indicated for patella replacement during TKA [12,19]. Furthermore, Longo et al. demonstrated in a detailed systematic review and meta-analysis that TKAs performed with patellar resurfacing have shown better outcomes than non-resurfaced TKAs, including significantly lower secondary operation and revision rates for the former group [20].

Yet, other studies have reached the opposite conclusion. Whiteside et al. found that the incidence of postoperative anterior knee pain was related to prosthesis design in patients with TKA retention, but there was no significant correlation with the degree of patellar cartilage degeneration [21]. Additionally, patellar resurfaced TKAs were associated with both greater anterior knee pain and a higher rate of revision in investigations by Pakos et al. and Calvisi et al. [5,22]. A systematic review conducted by Li et al. provided the conclusion that patellar resurfacing can reduce the risk of reoperation compared to TKA without resurfacing, but there is no evidence to suggest an improvement in postoperative knee function or patient satisfaction. Randomized controlled trials have been completed, but no conclusive evidence has been gathered concerning which option is superior [23-27].

There are several limitations to our study. Prior studies have shown that poor trajectory of the patella and the presence of painful afferent fibers in the soft tissue around the patella can also be responsible for knee pain, highlighting a potential confounding factor in this investigation [28]. To mitigate this possibility, electrosurgery was used around the patella to destroy the peripheral nerves and block neurogenic pain pathways. Still, of the seven patients with postoperative knee pain, four were due to poor patellar tracking, indicating a limitation of this study. Other shortcomings include the retrospective nature of this study and a lack of a control group to compare outcomes directly against TKAs performed with patellar resurfacing. A final area of weakness in the study design includes the fact that multiple surgeons conducted the surgeries, leading to varying operative techniques and post-operative rehabilitation protocols. In the future, as with many questions in orthopaedic surgery, high-quality, randomized controlled studies should be performed to best compare TKAs with versus without patellar resurfacing, and long-term efficacy needs to be followed over a longer postoperative time period.

\section{Conclusions}

The main finding of this study is that performing TKA without patellar resurfacing does not prohibit significant increases in postoperative functional outcome scores. These results indicate that satisfactory clinical outcomes can be achieved in patients with degenerative knee osteoarthritis but intact patellofemoral compartments when performing a TKA.

\section{Additional Information \\ Disclosures}

Human subjects: Consent was obtained or waived by all participants in this study. National Natural Science Foundation of China issued approval N/A. 1.1.2021 Dear "Cureus" Journal Editors: Our original article "Functional Outcomes Following Total Knee Arthroplasty Without Patellar Resurfacing: A Minimum 2-year Follow-Up Retrospective Cohort Study" previously titled "Patellar Resurfacing: Is it Needed?" was the original work performed by the research team of orthopedists at the Xinjian Medical University, Tumor Hospital, with additional affiliation to the First Affiliate Hospital and Second Affiliate hospital. All enrolled patients at these institutions have written, signed consent for inclusion in our prospectively collected, retrospectively reviewed database. These were ratified and approved by the Institutional Review Board of Ethic Committee of Xinjian Medical University in Ürümqi, the capital of the Xianjiang Uyghur Autonomous Region of the Peoples Republic of China. All patients at the orthopedic hospital are asked for written inclusion and at any time were given the ability to opt out at any time. All local and international regulations 
were adhered to per the National Natural Science Foundation of China. Sincerely yours, The Department of Orthopaedics, the First Affiliated Hospital, Xianjing Medical University, Ürümqi 830054, Xinjiang Uighur Autonomous Region, China Correspondence to: Hao Wen, Xinjiang Clinical Hydatid Research Institute and General Department, the First Affiliated Hospital of Xianjing Medical University, No. 1 LiYuShan Road, Ürümqi 830054, Xinjiang Uighur Autonomous Region, China. Telephone: +86-991-4363775 Fax: +86-9914324139. Animal subjects: All authors have confirmed that this study did not involve animal subjects or tissue. Conflicts of interest: In compliance with the ICMJE uniform disclosure form, all authors declare the following: Payment/services info: All authors have declared that no financial support was received from any organization for the submitted work. Financial relationships: All authors have declared that they have no financial relationships at present or within the previous three years with any organizations that might have an interest in the submitted work. Other relationships: All authors have declared that there are no other relationships or activities that could appear to have influenced the submitted work.

\section{References}

1. Meneghini RM: Should the patella be resurfaced in primary total knee arthroplasty? An evidence-based analysis. J Arthroplasty. 2008, 23:11-4. 10.1016/j.arth.2008.06.009

2. Ali A, Lindstrand A, Nilsdotter A, Sundberg M: Similar patient-reported outcomes and performance after total knee arthroplasty with or without patellar resurfacing. Acta Orthop. 2016, 87:274-9. 10.3109/17453674.2016.1170548

3. Maffulli N, Longo UG, Gougoulias N, Caine D, Denaro V: Sport injuries: a review of outcomes. Br Med Bull. 2011, 97:47-80. 10.1093/bmb/ldq026

4. Garneti N, Mahadeva D, Khalil A, McLaren CA: Patellar resurfacing versus no resurfacing in Scorpio total knee arthroplasty. J Knee Surg. 2008, 21:97-100. 10.1055/s-0030-1247802

5. Pakos EE, Ntzani EE, Trikalinos TA: Patellar resurfacing in total knee arthroplasty: a meta-analysis . J Bone Joint Surg Am. 2005, 87:1438-45. 10.2106/JBJS.D.02422

6. Meding JB, Fish MD, Berend ME, Ritter MA, Keating EM: Predicting patellar failure after total knee arthroplasty. Clin Orthop Relat Res. 2008, 466:2769-74. 10.1007/s11999-008-0417-y

7. Khan A, Pradhan N: Results of total knee replacement with/without resurfacing of the patella . Acta Ortop Bras. 2012, 20:300-2. 10.1590/S1413-78522012000500011

8. Aunan E, Næss G, Clarke-Jenssen J, Sandvik L, Kibsgård TJ: Patellar resurfacing in total knee arthroplasty: functional outcome differs with different outcome scores: A randomized, double-blind study of 129 knees with 3 years of follow-up. Acta Orthop. 2016, 87:158-64. 10.3109/17453674.2015.1111075

9. Parvizi J, Rapuri VR, Saleh KJ, Kuskowski MA, Sharkey PF, Mont MA: Failure to resurface the patella during total knee arthroplasty may result in more knee pain and secondary surgery. Clin Orthop Relat Res. 2005, 438:191-6. 10.1097/01.blo.0000166903.69075.8d

10. van Jonbergen HP, Barnaart AF, Verheyen CC: A Dutch survey on circumpatellar electrocautery in total knee arthroplasty. Open Orthop J. 2010, 4:201-3. 10.2174/1874325001004010201

11. Brick GW, Scott RD: The patellofemoral component of total knee arthroplasty . Clin Orthop Relat Res. 1988, 163-78.

12. Picetti GD 3rd, McGann WA, Welch RB: The patellofemoral joint after total knee arthroplasty without patellar resurfacing. J Bone Joint Surg Am. 1990, 72:1379-82.

13. Insall JN, Dorr LD, Scott RD, Scott WN: Rationale of the Knee Society clinical rating system . Clin Orthop Relat Res. 1989, 13-4.

14. Feller JA, Bartlett RJ, Lang DM: Patellar resurfacing versus retention in total knee arthroplasty . J Bone Joint Surg Br. 1996, 78:226-8.

15. Ewald FC: The Knee Society total knee arthroplasty roentgenographic evaluation and scoring system . Clin Orthop Relat Res. 1989, 9-12.

16. Lester JD, Watson JN, Hutchinson MR: Physical examination of the patellofemoral joint . Clin Sports Med. 2014, 33:403-12. 10.1016/j.csm.2014.03.002

17. Outerbridge RE: The etiology of chondromalacia patellae. J Bone Joint Surg Br. 1961, 43-B:752-7. 10.1302/0301-620X.43B4.752

18. Altay MA, Ertürk C, Altay N, Akmeșe R, Ișıkan UE: Patellar denervation in total knee arthroplasty without patellar resurfacing: a prospective, randomized controlled study. Orthop Traumatol Surg Res. 2012, 98:4215. 10.1016/i.otsr.2012.03.002

19. Rodríguez-Merchán EC, Gómez-Cardero P: The outerbridge classification predicts the need for patellar resurfacing in TKA. Clin Orthop Relat Res. 2010, 468:1254-7. 10.1007/s11999-009-1123-0

20. Longo UG, Ciuffreda M, Mannering N, D'Andrea V, Cimmino M, Denaro V: Patellar resurfacing in total knee arthroplasty: systematic review and meta-analysis. J Arthroplasty. 2018, 33:620-32. 10.1016/j.arth.2017.08.041

21. Whiteside LA, Nakamura T: Effect of femoral component design on unresurfaced patellas in knee arthroplasty. Clin Orthop Relat Res. 2003, 189-98. 10.1097/01.blo.0000063605.67412.4d

22. Calvisi V, Camillieri G, Lupparelli S: Resurfacing versus nonresurfacing the patella in total knee arthroplasty: a critical appraisal of the available evidence. Arch Orthop Trauma Surg. 2009, 129:1261-70. 10.1007/s00402-008-0801-9

23. Li S, Chen Y, Su W, Zhao J, He S, Luo X: Systematic review of patellar resurfacing in total knee arthroplasty . Int Orthop. 2011, 35:305-16. 10.1007/s00264-010-1109-2

24. Campbell DG, Duncan WW, Ashworth M, Mintz A, Stirling J, Wakefield L, Stevenson TM: Patellar resurfacing in total knee replacement: a ten-year randomised prospective trial. J Bone Joint Surg Br. 2006, 88:734-9. 10.1302/0301-620X.88B6.16822

25. Kajino A, Yoshino S, Kameyama S, Kohda M, Nagashima S: Comparison of the results of bilateral total knee arthroplasty with and without patellar replacement for rheumatoid arthritis: a follow-up note. J Bone Joint Surg Am. 1997, 79:570-4. 


\section{Cureus}

26. Mayman D, Bourne RB, Rorabeck CH, Vaz M, Kramer J: Resurfacing versus not resurfacing the patella in total knee arthroplasty: 8- to 10-year results. J Arthroplasty. 2003, 18:541-5. 10.1016/s0883-5403(03)00150-

27. Schroeder-Boersch H, Scheller G, Fischer J, Jani L: Advantages of patellar resurfacing in total knee arthroplasty: two-year results of a prospective randomized study. Arch Orthop Trauma Surg. 1998, 117:738. 10.1007/BF00703446

28. Breugem SJ, Haverkamp D: Anterior knee pain after a total knee arthroplasty: What can cause this pain? World J Orthop. 2014, 5:163-70. 10.5312/wjo.v5.i3.163 